\title{
Reducing ambiguity in lotteries: evidence from a field experiment
}

\author{
Julian Conrads, Tommaso Reggiani \& Rainer Michael Rilke
}

To cite this article: Julian Conrads, Tommaso Reggiani \& Rainer Michael Rilke (2016) Reducing ambiguity in lotteries: evidence from a field experiment, Applied Economics Letters, 23:3, 206-211, DOI: $10.1080 / 13504851.2015 .1066480$

To link to this article: http://dx.doi.org/10.1080/13504851.2015.1066480

Published online: 11 Aug 2015.

Submit your article to this journal \lceil

W Article views: 48

Q View related articles $\sqsubset$

View Crossmark data $₫$ 


\title{
Reducing ambiguity in lotteries: evidence from a field experiment
}

\author{
Julian Conrads ${ }^{\mathrm{a}}$, Tommaso Reggiani $\mathbb{1}^{\mathrm{b}}$ and Rainer Michael Rilke \\ aDepartment of Corporate Development and Business Ethics, University of Cologne, Cologne, Germany; ${ }^{b}$ DFG Research group 'Design and \\ Behavior' \& Department of Corporate Development and Business Ethics, University of Cologne, Cologne, Germany
}

ABSTRACT

Ambiguity about the chances of winning represents a key aspect in lotteries. By means of a controlled field experiment, we exogenously vary the degree of ambiguity about the winning chances of lotteries organized to incentivize the contribution for a public good. In one treatment, people have been simply informed about the maximum number of potential participants (i.e. the number of lottery tickets released). In a second treatment, this information has been omitted as in all traditional lotteries. Our general finding shows that simply reducing the degree of ambiguity of the lottery leads to a sizable and significant increase $(67 \%)$ in the participation rate. This result is robust to alternative prize configurations.
"That knowing is better than wondering"

(Benjamin Franklin)

\section{Introduction}

Financing public goods or charitable projects by lotteries outperforms voluntary contribution mechanisms (VCM), i.e. more people contribute when simultaneously participating in a lottery compared to just asking for a voluntary contribution. Therefore, practitioners, e.g. governments, charities or civic groups, make extensive use of lotteries to achieve higher levels of contribution in public goods (e.g. Lange, List, and Price 2007). Also firms might want to exploit lotteries, to get high response rates in conducting surveys with their customers or personnel. ${ }^{1}$ This mechanism of using lotteries is theoretically developed in the seminal paper by Morgan (2000) and empirically corroborated by experimental studies (e.g. Morgan and Sefton 2000).

A crucial feature about the attractiveness of lotteries relates to the knowledge potential participants have about the number of other possible participants since it affects a participant's likelihood to win. In general, people prefer events they know more about, i.e. low ambiguity settings, over situations that are uncertain of, i.e. high ambiguity settings (see Ellsberg 1963, who initiated this research, and Camerer and Weber 1992, for a review).

The aim of this article is to experimentally analyse the effects on subjects' propensity to voluntarily participate in a scientific survey when incentivized with lotteries characterized by different degrees of ambiguity about the number of possible participants in this lottery.

In the laboratory experiment by Lange, List, and Price (2007), participants know the exact number of other group members that can potentially win a lottery prize when contributing to a public good. Hence, this environment is characterized by the lowest degree of ambiguity or, more specifically, the authors study a risk environment. By contrast, in the field experimental study by Landry et al. (2006) participants are only informed that they will participate in a lottery against an unknown number of other households when they contribute to a public good. Both studies show that contributions to public goods increase when a lottery is offered; however, they do not compare whether contributions differ with respect to different degrees of ambiguity about chances of winning the lottery.

CONTACT: Tommaso Reggiani tommaso.reggiani@uni-koeln.de

${ }^{1}$ Another way to motivate customers to complete a survey is analysed by Gneezy and Rey-Biel (2014) who compare contingent with noncontingent incentives.

๑) 2015 Taylor \& Francis 
The aim of our field experiment is to analyse whether the contribution rates to a public good increase by decreasing the ambiguity about the chances to win a lottery for contributing. As the contribution to a public good, subjects are asked to participate in an online survey for scientific reasons. As an incentive to fill in the online survey, subjects are either offered lottery tickets with High Ambiguity (HA) or Low Ambiguity (LA) about the chances to actually win the lottery. In the high ambiguity treatment, subjects have no information about the number of potential participants. In the low ambiguity treatment, subjects are informed about the number of potential participants who are asked to fill in the online survey (but not about the number of actual participants). Contrasting the participation rates in the two main treatments, we find that reducing the degree of ambiguity about the chances to win significantly increases by $67 \%$ the number of subjects filling in the online survey. This finding also remains robust comparing two different systems of lottery prizes, i.e. comparing a single large prize with a multiple small prize lottery (see also the experimental design of Lange, List, and Price 2007).

The remainder of the article is organized as follows: In the next section, the experimental design is presented. The results are presented in the 'Results' section. The results are discussed and concluded in the 'Conclusion and discussion' section.

\section{Experimental design}

In order to conduct an online survey, ${ }^{2} 2467$ invitation emails were sent out. Aiming to foster the participation rate in the survey (i.e. promoting the contribution to this specific kind of public good represented by data collection for scientific purposes), different lotteries - each of which yielded up to 400 Euros - were linked to the survey. Upon survey completion, each subject was awarded a single lottery ticket. ${ }^{3}$ The pool of subjects consisted of students from the University of Cologne, Faculty of Economics, Management and Social Science. Among
Table 1. Experimental treatments and number of observations.

\begin{tabular}{lcc}
\hline & \multicolumn{2}{c}{ Treatments } \\
\cline { 2 - 3 } & High ambiguity & Low ambiguity \\
\hline Single-Large prize & $589(23.88 \%)$ & $633(25.66 \%)$ \\
Multiple-Small prizes & $610(24.73 \%)$ & $635(25.74 \%)$ \\
Pooled & $1199(48.60 \%)$ & $1268(51.40 \%)$ \\
\hline
\end{tabular}

2467 subjects, $46.17 \%$ were females. Subjects were randomized across lotteries treatments.

The between-subjects $2 \times 2$ lottery experimental design consists of two main treatments, i.e. High Ambiguity (HA) versus Low Ambiguity (LA), combined with two different - but equivalent - rewarding configurations, i.e. Single-Large prize $(S P)$ versus Multiple-Small prizes (MP), in order to check if the effects generated by High versus Low Ambiguity are robust given alternative rewarding configurations (see Table 1).

\section{Main experimental manipulation: high ambiguity versus low ambiguity}

On 6 November 2012, 589 invitation emails in the High Ambiguity (HA/SP) experimental condition were randomly sent out. Under this treatment featured by total ambiguity, (1) it WAS NOT mentioned that the same invitation was sent to 589 students in total. It was clearly stated (2) that upon participation in the survey, each subject would have one chance of winning one single prize yielding 400 Euros and (3) that the deadline for participating in the survey was 30 November 2012.

In order to prevent contagion between the two main treatments featured by different degrees of ambiguity, we ran a Low Ambiguity (LA/SP) treatment 1 year later addressing the very same population of subjects (of course excluding all students approached the year before for the HA/SP treatment).

On 6 December 2013, 633 new invitation emails in the Low Ambiguity (LA/SP) experimental condition were randomly sent out. ${ }^{4}$ Differently from the previous case, the current lottery is distinguished by partial ambiguity since (1) it WAS clearly communicated that the same invitation was sent to 633

\footnotetext{
${ }^{2}$ Aside from the indication of demographics, the survey contains questions on personal values (Schwartz 1992) and on possible motives to volunteer (Clary et al. 1998).

${ }^{3}$ In this practical case, the effort exerted in order to fill in the survey represents the price of the lottery ticket. Since the hyperlinks to access the survey were individualized for each different invitation, it was not possible to fill in the online survey more than once in order to increase the probability of winning. For this reason this lottery can be considered a raffle, see Morgan (2000).

${ }^{4}$ Excluding from the pool all subjects approached for the previous HA/SP lottery.
} 
students in total. Like in the other treatment, it was stated (2) that upon participation in the survey, each subject would have one chance of winning one single prize yielding 400 Euros and (3) that the deadline for participating in the survey was 30 December 2013.

\section{Robustness test: single reward versus multiple rewards}

In addition to the main comparative evaluation described above, a further field study was devised to check if results might be sensitive to the configuration of the reward scheme. The two treatments High Ambiguity versus Low Ambiguity were then replicated with the only difference that now, upon participation in the survey, each subject has the chance of winning one out of eight prizes yielding 50 Euros each (MP configuration) instead of one single prize of 400 Euros (SP configuration). Note that the total size of the lottery reward is constant under the two alternative reward configurations (Single-Large prize versus Multiple-Small prizes).

On 6 November 2012, 610 invitation emails in the High Ambiguity (HA/MP) treatment were randomly sent out. ${ }^{5}$ As before, under this treatment featured by a total ambiguity, (1) it WAS NOT mentioned that the same invitation was sent to 610 students in total. It was clearly stated (2) that upon participation in the survey, each subject would have one chance of winning one out of the eight prizes of 50 Euros and (3) that the deadline for participating in the survey was 30 November 2012.

Also in this second case, in order to avoid any contagion between treatments featured by different degrees of ambiguity, we ran the Low Ambiguity/MP treatment 1 year later (in 2013) addressing the very same population of subjects (of course excluding all students approached the year before for the $H A / M P$ treatment).

On 6 December 2013, 635 new invitation emails in the Low Ambiguity (LA/MP) treatment were sent out. $^{6}$ Differently from the homologous High Ambiguity $(H A / M P)$ case, now the lottery features partial ambiguity since (1) it WAS clearly communicated that the same invitation was sent to 635 students in total. Like in the other treatment, it was stated (2) that upon participation in the survey, each subject would have one chance of winning one out of eight prizes yielding 50 Euros and (3) that the deadline for participating in the survey was 30 December 2013.

We prioritized minimizing the risk of contagion between treatments marked by different degrees of ambiguity (High in 2012, Low in 2013) ahead of the risk of spillovers between the two reward configurations (Single-Large versus Multiple-Small) following well-defined considerations. First of all, our experimental manipulation mainly focuses on the different degrees of ambiguity found in the lotteries, while the reward configuration represents only a side experimental variation in order to validate the robustness of the effects caused by ambiguity manipulations. Second, the two alternative rewarding configurations are monetarily equivalent (400 Euros versus $8 \times 50$ Euros $=400$ Euros). ${ }^{7}$

\section{Results}

The analysis of our results is structured around our main research question: how does the degree of ambiguity influence voluntary participation in our lottery? In order to do this, we will focus on the number of questionnaires that have been completed by our subjects, i.e. the participation rates. In a first step, we will consider the participation rates between the different levels of ambiguity. In a second step, we will contrast the participation rate between the different price configurations.

\section{Ambiguity reduction fosters agents' participation}

Considering first the baseline Single-Large prize configuration under the two different ambiguity treatments, we observe (see Table 2 and Fig. 1) the average participation to be $21.96 \%$ under the Low Ambiguity (LA/SP) treatment but only $14.26 \%$ under High Ambiguity (HA/SP). The relative performance increase of $57 \%$ is economically

\footnotetext{
${ }^{5}$ Excluding from the pool all subjects already involved in the contemporaneous HA/SP lottery (see Table 2).

${ }^{6}$ Excluding from the pool both subjects involved in the previous HA/SP and HA/MP lotteries and the ones exposed to the contemporaneous $L A / S P$ treatment (see Table 2).

${ }^{7}$ We received no complaints or requests of clarification either during the field phase of the experiments or after the assignment of the lotteries rewards.
} 
Table 2. Participation rates by Treatments/Configurations.

\begin{tabular}{lccc}
\hline & \multicolumn{3}{c}{ Treatments } \\
\cline { 2 - 4 } & High ambiguity & Low ambiguity \\
\hline Single-Large prize & $14.26 \%(84 / 589)$ & $(* * *)$ & $21.96 \%(139 / 633)$ \\
& $(* *)$ & $<$ & $(=)$ \\
& $\checkmark$ & & $\vee$ \\
Multiple-Small prizes & $10.16 \%(62 / 610)$ & $(* * *)$ & $18.90 \%(121 / 635)$ \\
Pooled & $145 / 1199(12.09 \%)$ & $(* * *)$ & $259 / 1268(20.43 \%)$ \\
& & $<$ &
\end{tabular}

Note: The asterisks display the statistical significance levels of two-sided Fisher's exact test: $*=10 \%$ level, $* *=5 \%$ level, $* * *=1 \%$ level.

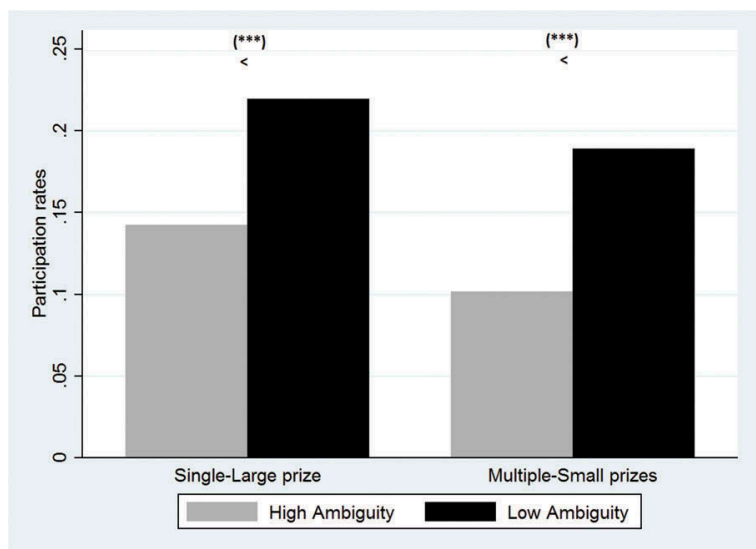

Figure 1. Participation rates by Treatment (conditional to Configuration).

meaningful in its size and highly statistically significant according to a two-sided Fisher's exact test $(p<0.001)$.

The pattern is reproduced when an equivalent Multiple-Small prizes configuration is implemented. In this case, the participation rate is $18.90 \%$ under the Low Ambiguity (LA/MP) treatment but only $10.16 \%$ under High Ambiguity (HA/MP). Also in this case, the relative difference in performance of almost $90 \%$ is economically meaningful in its size and highly statistically significant according to a two-sided Fisher's exact test $(p<0.001)$.

As a consequence of this clear pattern, at aggregate level polling together the two configurations SingleLarge Prize and Multiple-Small Prizes by treatments, under the Low Ambiguity treatment, the average rate of participation in the survey is $20.5 \%$, but it halves to $12 \%$ under High Ambiguity (see Table 2). The 67\% increase in relative performance, generated simply by lowering the degree of ambiguity of the lottery, proved to be economically significant in its magnitude and highly statistically significant according to a two-sided Fisher's exact test $(p<0.001)$.

\section{Ambiguity reduction promotes rational choice}

Since the two alternative reward configurations are monetarily equivalent ( $S P$ : 400 Euros versus $M P$ : $8 \times 50$ Euros $=400$ Euros), conditional to a given degree of ambiguity of the lottery ( $H A$ versus $L A$ ), no differences in participation rates should emerge between the alternative reward configurations.

If we look at the High Ambiguity treatment (see Table 2 and Fig. 2), while the participation rate is $14.26 \%$ under the Single-Large prize condition it declines to $10.16 \%$ under the Multiple-Small prizes condition. This difference is statistically significant at the $5 \%$ level ( $p=0.034$ ) according to a two-sided Fisher's exact test.

On the contrary, under the Low Ambiguity treatment the participation rates prove to be statistically indistinguishable. While the participation rate is $21.96 \%$ under the Single-Large prize treatment, it declines marginally to $18.90 \%$ under the MultipleSmall prizes condition. The relative magnitude of the effect is small (15\%) and not statistically significant at any conventional level $(p=0.186)$. Simply providing information about the maximum number of potential participants (Low Ambiguity manipulation) leads a Multiple-Small prizes lottery to converge to the performance level of a more salient ${ }^{8}$ Single-Large prize one. This shows how simple information concerning the 'number of ticked issued' (number of

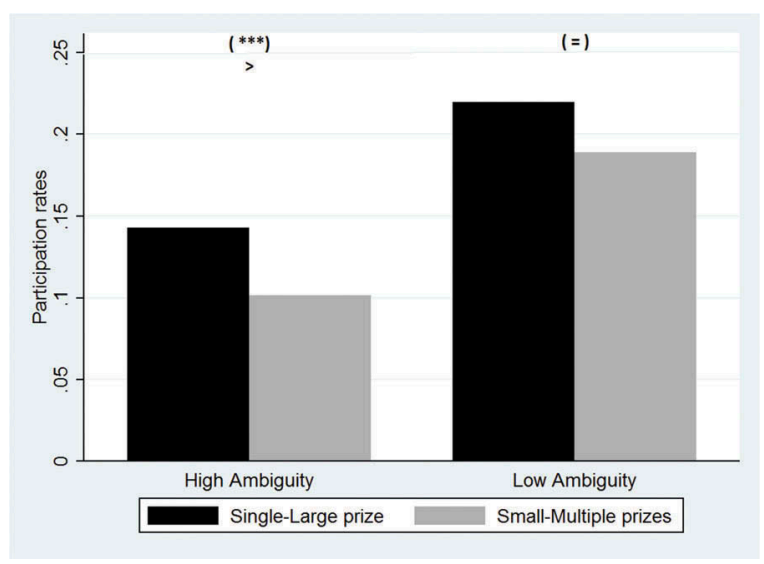

Figure 2. Participation rates by Configuration (conditional to Treatment).

\footnotetext{
${ }^{8}$ About the relative lower performance of multiple prizes lotteries/auctions, see Faravelli and Stanca (2012), Lange, List, and Price (2007) and Landry et al.
} (2006). 
potential participants in the survey in our case) can substantially contribute to an improvement in rational agents' decision-making.

\section{Conclusion and discussion}

By means of a controlled field experiment, we investigated how ambiguity concerns affect agents' participation into lotteries designed to promote agents' contribution to a public good. In one treatment distinguished by Low Ambiguity, we exogenously manipulated the degree of ambiguity of the lottery by informing all invited participants about the maximum number of potential participants (i.e. the number of lottery tickets released). In a second treatment characterized by High Ambiguity, this information was omitted as in standard traditional lotteries.

Our general finding shows that by simply reducing the degree of ambiguity of the lottery by announcing the maximum number of potential participants, the participation rate of the lottery increases significantly by $67 \%$. This result proves to be robust to alternative rewarding configurations of the lottery based on Single-Large prize and MultipleSmall prizes, respectively.

Our findings are based on medium-scale lotteries involving approximately 600 potential participants. Further research is needed in order to study the effect of varying the number of tickets released and announced between small and very large amounts. It might be the case that for very large-scale lotteries, such as the nationwide ones, the announcement of an extremely large number of tickets or potential participants could discourage participation compared with a fully ambiguous lottery.

\section{Acknowledgements}

We thank Louis Eeckhoudt, Felix Kölle, Minkia Martiale and Sarah Grace See for comments and suggestions.

\section{Disclosure statement}

No potential conflict of interest was reported by the authors.

\section{Funding}

Financial support from the Deutsche Forschungsgemeinschaft through grant 'TP3 Design of Incentive Schemes within Firms: Bonus Systems and Performance Evaluation' (sub-project of the DFG-Forschergruppe 'Design and Behavior') and through the Leibniz-Award to Axel Ockenfels is gratefully acknowledged.

\section{ORCID}

Tommaso Reggiani (D) http://orcid.org/0000-0002-3134-1049

\section{References}

Camerer, C., and M. Weber. 1992. "Recent Developments in Modeling Preferences: Uncertainty and Ambiguity." Journal of Risk and Uncertainty 5 (4): 325-370. doi:10.1007/BF00122575.

Clary, E. G., M. Snyder, R. D. Ridge, J. Copeland, A. A. Stukas, J. Haugen, P. Miene, et al. 1998. "Understanding and Assessing the Motivations of Volunteers: A Functional Approach." Journal of Personality and Social Psychology 74: 1516-1530. doi:10.1037/00223514.74.6.1516.

Ellsberg, D. 1963. "[Risk, Ambiguity, and the Savage Axioms]: Reply." The Quarterly Journal of Economics 77: 336-342. doi:10.2307/1884409.

Faravelli, M., and Stanca, L. 2012. "Single Versus Multiple Prize Contests to Finance Public Goods: Theory and Experimental Evidence." Journal of Economic Behavior and Organization 81: 677-688. http://www.sciencedirect.com/science/article/ pii/S0167268111002563

Gneezy, U., and P. Rey-Biel. 2014. "On the Relative Efficiency of Performance Pay and Noncontingent Incentives." Journal of the European Economic Association 12 (1): 62-72. doi:10.1111/jeea.12062.

Landry, C. E., A. Lange, J. A. List, M. K. Price, and N. G. Rupp. 2006. "Toward an Understanding of the Economics of Charity: Evidence from a Field Experiment." The Quarterly Journal of Economics 121 (2): 747-782. doi:10.1162/qjec.2006.121.2.747.

Lange, A., J. A. List, and M. K. Price. 2007. "Using Lotteries to Finance Public Goods: Theory and Experimental Evidence." International Economic Review 48 (3): 901927. doi:10.1111/iere.2007.48.issue-3.

Morgan, J. 2000. "Financing Public Goods by Means of Lotteries." The Review of Economic Studies 67 (4): $761-$ 784. doi:10.1111/1467-937X.00153.

Morgan, J., and M. Sefton. 2000. "Funding Public Goods with Lotteries: Experimental Evidence." The Review of Economic Studies 67 (4): 785-810. doi:10.1111/1467937X.00154.

Schwartz, S. H. 1992. "Universals in the Content and Structure of Values: Theoretical Advances and Empirical tests in 20 Countries." Advances in Experimental Social Psychology 25 (1): 1-65. 


\section{Appendix}

\section{Invitation email translated from German ${ }^{a}$ :} Cologne, \{HA: 06/11/2012\}/\{LA: 06/12/2013\}

Dear student,

For a research project of the University of Cologne, we are conducting an online survey among students of the Business and Economics Faculty. -The completion of the survey will take 10 minutes of your time and is anonymous. You have time until \{HA: 06/11/ $2012\} /\{L A: 06 / 12 / 2013\}$. -In order to incentivize your voluntary participation, the survey is linked to a lottery.

$\{S P$ : Among all the actual participants of the survey, we draw 1 participant. He/she wins $€ 400$.
\{MP: Among all the actual participants of the survey, we draw 8 different participants. They win $€ 50$ each. $\}$

$\{L A:$ This invitation is sent out to $\{M P: 633\} /$ \{SP: 635 $\}$ potential participants. $\}$

$\{H A:-\}$

The outcome the lottery will be disclosed via private email.

You reach the survey via the following link: < \#online-link\# > Thank you for your participation!

Your answers are of great value to us.

Kind regards,

The research team

${ }^{a}$ Abbreviations. $H A$ : High Ambiguity treatment; LA: Low Ambiguity treatment; $S P$ : Single-Large prize configuration; $M P$ : Multiple-Small prizes configuration. 